Elect. Comm. in Probab. 15 (2010), 457-474

\title{
EXPLICIT SOLUTIONS TO FRACTIONAL DIFFUSION EQUATIONS VIA GENERALIZED GAMMA CONVOLUTION
}

\author{
MIRKO D'OVIDIO \\ Department of Statistics, Probability and Applied Statistics. Sapienza University of Rome, Ple Aldo \\ Moro, 5 - 00185 Rome (Italy). \\ email: mirko.dovidio@uniroma1.it
}

Submitted April 18, 2010, accepted in final form September 20, 2010

AMS 2000 Subject classification: 60J65, 60J60, 26A33

Keywords: Mellin convolution formula, generalized Gamma r.v.'s, Stable subordinators, Fox functions, Bessel processes, Modified Bessel functions.

\begin{abstract}
In this paper we deal with Mellin convolution of generalized Gamma densities which leads to integrals of modified Bessel functions of the second kind. Such convolutions allow us to explicitly write the solutions of the time-fractional diffusion equations involving the adjoint operators of a square Bessel process and a Bessel process.
\end{abstract}

\section{Introduction and main result}

In the last years, the analysis of the compositions of processes and the corresponding governing equations has received the attention of many researchers. Many of them are interested in compositions involving subordinators, in other words, subordinated processes $Y(T(t)$ ), $t>0$ (according to [9]) where $T(t), t>0$ is a random time with non-negative, independent and homogeneous increments (see [4]). If the random time is a (symmetric or totally skewed) stable process we have results which are strictly related to the Bochner's subordination and the p.d.e.'s connections have been investigated, e.g., in $[6 ; 7 ; 8 ; 22 ; 23]$. If the random time is an inverse stable subordinator we shall refer to the governing equation of $Y(T(t))$ as a fractional equation considering that a fractional time-derivative must be taken into account. In the literature, several authors have studied the solutions to space-time fractional equations. In the papers by Wyss [30], Schneider and Wyss [29], the authors present solutions of the fractional diffusion equation $\partial_{t}^{\lambda} T=\partial_{x}^{2} T$ in terms of Fox's functions (see Section 2). In the works by Mainardi et al., see e.g. $[17 ; 18]$ the authors have shown that the solutions to space-time fractional equation ${ }_{x} D_{\theta}^{\alpha} u={ }_{t} D_{*}^{\beta} u$ can be represented by means of Mellin-Barnes integral representations (or Fox's functions) and M-Wright functions (see e.g. Kilbas et al. [13]). The fractional Cauchy problem $D_{t}^{\alpha} u=L u$ has been thoroughly studied by yet other authors and several representations of the solutions have been carried out, but an explicit form of the solutions has never been obtained. Nigmatullin [25] gave a physical derivation when $L$ is the generator of some continuous Markov process. Zaslavsky [31] introduced the space-time fractional kinetic equation for Hamiltonian chaos. Kochubei $[14,15]$ first introduced a mathemat- 
ical approach while Baeumer and Meerschaert [1] established the connections between fractional problem and subordination by means of inverse stable subordinator when $L$ is an infinitely divisible generator on a finite dimensional vector space. In particular, if $\partial_{t} p=L p$ is the governing equation of $\mathbf{X}(t)$, then under certain conditions, $\partial_{t}^{\beta} q=L q+\delta(\mathbf{x}) t^{-\beta} / \Gamma(1-\beta)$ is the equation governing the process $\mathbf{X}\left(V_{t}\right)$ where $V_{t}$ is the inverse or hitting time process to the $\beta$-stable subordinator, $\beta \in(0,1)$. Orsingher and Beghin [26, 27] found explicit representations of the solutions to $\partial_{t}^{v} u=\lambda^{2} \partial_{x}^{2} u$ only in some particlular cases: $v=(1 / 2)^{n}, 1 / n, n \in \mathbb{N}$ and $v=1 / 3,2 / 3,4 / 3$. Also, they represented the solutions to the fractional telegraph equations in terms of stable densities, see $[3 ; 26]$. In general, the solutions to fractional equations represent the probability densities of certain subordinated processes obtained by using a time clock (in the following we will refer to it as $L_{t}^{v}$ ) which is an inverse stable subordinator (see Section 4). For a short review on this field, see also Nane [24] and the references therein.

We will present the role of the Mellin convolution formula in finding solutions of fractional diffusion equations. In particular, our result allows us to write the distribution of both stable subordinator and its inverse process whose governing equations are respectively space-fractional or time-fractional equations. This result turns out to be useful for representing the solutions to the following fractional diffusion equation

$$
D_{t}^{v} \tilde{u}_{v}^{\gamma, \mu}=\mathscr{G}_{\gamma, \mu} \tilde{u}_{v}^{\gamma, \mu}
$$

where $\tilde{u}_{v}^{\gamma, \mu}=\tilde{u}_{v}^{\gamma, \mu}(x, t), x>0, t>0, D_{t}^{v}$ is the Riemann-Liouville fractional derivative, $v \in(0,1]$ and $\mathscr{G}_{\gamma, \mu}$ is an operator to be defined below (see formula (3.4)). We present, for $v=1 /(2 n+1)$, $n \in \mathbb{N} \cup\{0\}$, the explicit solutions to (1.1) in terms of integrals of modified Bessel functions of the second kind $\left(K_{v}\right)$ whereas, for $v \in(0,1]$, we obtain the solutions to (1.1) in terms of Fox's functions. After some preliminaries in Section 2, in Section 3 we recall the generalized Gamma density $Q_{\mu}^{\gamma}$ starting from which we define the distribution $g_{\mu}^{\gamma}$ of the (generalized Gamma) process $G_{t}^{\gamma, \mu}$ and the distribution $e_{\mu}^{\gamma}$ of the process $E_{t}^{\gamma, \mu}$. The latter can be seen as the reciprocal Gamma process, indeed $E_{t}^{\gamma, \mu}=1 / G_{t}^{\gamma, \mu}$, or in a more striking interpretation, as the hitting time process for which $\left(E_{t}^{\gamma, \mu}<x\right)=\left(G_{x}^{\gamma, \mu}>t\right)$. We shall refer to $E_{t}^{\gamma, \mu}$ as the reciprocal or equivalently the inverse process of $G_{t}^{\gamma, \mu}$. It must be noticed that $e_{\mu}^{\gamma}=g_{\mu}^{-\gamma}$ because $G_{t}^{-\gamma, \mu}=1 / G_{t}^{\gamma, \mu}$. Furthermore, we introduce the most important tool we deal with in this paper, the Mellin convolutions $g_{\bar{\mu}}^{\gamma, \star n}$ (see formula (3.14) ) and $e_{\bar{\mu}}^{\star n}$ (see formula (3.13) ) where $e_{\bar{\mu}}^{\star n}$ stands for $e_{\bar{\mu}}^{1, \star n}$. In Section 4 we draw some useful transforms of the distribution $h_{v}$ of the stable subordinator $\tilde{\tau}_{t}^{v}$ and the distribution $l_{v}$ of the inverse process $L_{t}^{v}$. Similar calculations can be found in the paper by Schneider and Wyss [29]. The inverse (or hitting time) process is defined once again from the fact that $\left(L_{t}^{v}<x\right)=\left(\tilde{\tau}_{x}^{v}>t\right)$ (see also [1; 4]). In Section 5 we present our main contribution. We show that the following representations hold true:

$$
h_{v}(x, t)=e_{\bar{\mu}}^{\star n}\left(x, \varphi_{n+1}(t)\right), \quad x>0, t>0, v=1 /(n+1), n \in \mathbb{N}
$$

and

$$
l_{v}(x, t)=g_{\bar{\mu}}^{(n+1), \star n}\left(x, \psi_{n+1}(t)\right), \quad x>0, t>0, v=1 /(n+1), n \in \mathbb{N} .
$$

where $\bar{\mu}=\left(\mu_{1}, \ldots, \mu_{n}\right), \mu_{j}=j v, j=1,2, \ldots, n, v=1 /(n+1), n \in \mathbb{N}$ and the time-stetching functions are given by $\varphi_{m}(s)=(s / m)^{m}$ and $\psi_{m}(s)=m s^{1 / m}, s \in(0, \infty), m \in \mathbb{N}, \psi=\varphi^{-1}$. The discussion made so far allows us to introduce the result stated in Theorem 1 . For $v=1 /(n+1)$, 
$n \in \mathbb{N} \cup\{0\}$, the solutions to (1.1) can be written as follows

$$
\tilde{u}_{v}^{\gamma, \mu}(x, t)=\int_{0}^{\infty} g_{\mu}^{\gamma}\left(x, s^{1 / \gamma}\right) g_{\bar{\mu}}^{1 / v, \star(1 / v-1)}\left(s, \psi_{1 / v}(t)\right) d s, \quad x \in(0, \infty), t>0
$$

where, for $n \in 2 \mathbb{N} \cup\{0\}$, we have

$$
g_{\bar{\mu}}^{1 / v, \star(1 / v-1)}(x, t)=\frac{1}{v^{1 / 2 v}}\left(\frac{x}{\pi^{2} t^{3}}\right)^{\frac{1-v}{4 v}} \int_{0}^{\infty} \ldots \int_{0}^{\infty} \mathscr{Q}_{\frac{1-v}{2}}\left(x, s_{1}\right) \ldots \mathscr{Q}_{\frac{1-v}{2}}\left(s_{n-1}, t\right) d s_{1} \ldots d s_{n-1}
$$

and

$$
\mathscr{Q}_{\frac{1-v}{2}}(x, t)=K_{\frac{1-v}{2}}\left(2 \sqrt{(x / t)^{1 / v}}\right), \quad x>0, t>0 .
$$

As a direct consequence of this result we obtain $\tilde{u}_{1}^{\gamma, \mu}=\tilde{g}_{\mu}^{\gamma}$, for $v=1$, where $\tilde{g}_{\mu}^{\gamma}(x, t)=g_{\mu}^{\gamma}\left(x, t^{1 / \gamma}\right)$ and the governing equation writes

$$
\frac{\partial}{\partial t} \tilde{u}_{1}^{\gamma, \mu}=\frac{1}{\gamma^{2}}\left(\frac{\partial}{\partial x} x^{2-\gamma} \frac{\partial}{\partial x}-(\gamma \mu-1) \frac{\partial}{\partial x} x^{1-\gamma}\right) \tilde{u}_{1}^{\gamma, \mu}, \quad x>0, t>0 .
$$

Furthermore, for $\gamma=1,2$ and $v \in(0,1]$ we obtain

$$
D_{t}^{v} \tilde{u}_{v}^{1, \mu}=\left(x \frac{\partial^{2}}{\partial x^{2}}-(\mu-2) \frac{\partial}{\partial x}\right) \tilde{u}_{v}^{1, \mu}, \quad x>0, t>0, \mu>0
$$

and

$$
D_{t}^{v} \tilde{u}_{v}^{2, \mu}=\frac{1}{2^{2}}\left(\frac{\partial^{2}}{\partial x^{2}}-\frac{\partial}{\partial x} \frac{(2 \mu-1)}{x}\right) \tilde{u}_{v}^{2, \mu}, \quad x>0, t>0, \mu>0 .
$$

Equation (1.3) represents a fractional diffusion around spherical objects and thus, the solutions we deal with obey radial diffusion equations.

\section{Preliminaries}

The $\mathrm{H}$ functions were introduced by Fox [10] in 1996 as a very general class of functions. For our purpose, the Fox's $\mathrm{H}$ functions will be introduced as the class of functions uniquely identified by their Mellin transforms. A function $f$ for which the following Mellin transform exists

$$
\mathscr{M}[f(\cdot)](\eta)=\int_{0}^{\infty} x^{\eta} f(x) \frac{d x}{x}, \quad \Re\{\eta\}>0
$$

can be written in terms of $\mathrm{H}$ functions by observing that

$$
\int_{0}^{\infty} x^{\eta} H_{p, q}^{m, n}\left[x \mid \begin{array}{l}
\left(a_{i}, \alpha_{i}\right)_{i=1, \ldots, p} \\
\left(b_{j}, \beta_{j}\right)_{j=1, \ldots, q}
\end{array}\right] \frac{d x}{x}=\mathscr{M}_{p, q}^{m, n}(\eta), \quad \Re\{\eta\} \in \mathscr{D}
$$

where

$$
\mathscr{M}_{p, q}^{m, n}(\eta)=\frac{\prod_{j=1}^{m} \Gamma\left(b_{j}+\eta \beta_{j}\right) \prod_{i=1}^{n} \Gamma\left(1-a_{i}-\eta \alpha_{i}\right)}{\prod_{j=m+1}^{q} \Gamma\left(1-b_{j}-\eta \beta_{j}\right) \prod_{i=n+1}^{p} \Gamma\left(a_{i}+\eta \alpha_{i}\right)}
$$


The inverse Mellin transform is defined as

$$
f(x)=\frac{1}{2 \pi i} \int_{\theta-i \infty}^{\theta+i \infty} \mathscr{M}[f(\cdot)](\eta) x^{-\eta} d \eta
$$

at all points $x$ where $f$ is continuous and for some real $\theta$. Thus, according to a standard notation, the Fox $\mathrm{H}$ function is defined as follows

$$
H_{p, q}^{m, n}\left[\begin{array}{l|l}
x & \left(a_{i}, \alpha_{i}\right)_{i=1, \ldots, p} \\
\left(b_{j}, \beta_{j}\right)_{j=1, \ldots, q}
\end{array}\right]=\frac{1}{2 \pi i} \int_{\mathbb{P}(\mathscr{D})} \mathscr{M}_{p, q}^{m, n}(\eta) x^{-\eta} d \eta
$$

where $\mathbb{P}(\mathscr{D})$ is a suitable path in the complex plane $\mathbb{C}$ depending on the fundamental strip $(\mathscr{D})$ such that the integral (2.1) converges. For an extensive discussion on this function see Fox [10]; Mathai and Saxena [20]. The Mellin convolution formula

$$
f_{1} \star f_{2}(x)=\int_{0}^{\infty} f_{1}(x / s) f_{2}(s) \frac{d s}{s}, \quad x>0
$$

turns out to be very useful later on. Formula (2.3) is a convolution in the sense that

$$
\mathscr{M}\left[f_{1} \star f_{2}(\cdot)\right](\eta)=\mathscr{M}\left[f_{1}(\cdot)\right](\eta) \times \mathscr{M}\left[f_{2}(\cdot)\right](\eta) .
$$

Throughout the paper we will consider the integral

$$
f_{1} \circ f_{2}(x, t)=\int_{0}^{\infty} f_{1}(x, s) f_{2}(s, t) d s
$$

(for some well-defined $f_{1}, f_{2}$ ) which is not, in general, a Mellin convolution. We recall the following connections between Mellin transform and both integer and fractional order derivatives. In particular, we consider a rapidly decreasing function $f:[0, \infty) \mapsto[0, \infty)$, if there exists $a \in \mathbb{R}$ such that

$$
\lim _{x \rightarrow 0^{+}} x^{a-k-1} \frac{d^{k}}{d x^{k}} f(x)=0, \quad k=0,1, \ldots, n-1, \quad n \in \mathbb{N}, x \in \mathbb{R}_{+}
$$

then we have

$$
\mathscr{M}\left[\frac{d^{n}}{d x^{n}} f(\cdot)\right](\eta)=(-1)^{n} \frac{\Gamma(\eta)}{\Gamma(\eta-n)} \mathscr{M}[f(\cdot)](\eta-n)
$$

and, for $0<\alpha<1$

$$
\mathscr{M}\left[\frac{d^{\alpha}}{d x^{\alpha}} f(\cdot)\right](\eta)=\frac{\Gamma(\eta)}{\Gamma(\eta-\alpha)} \mathscr{M}[f(\cdot)](\eta-\alpha)
$$

(see Kilbas et al. [13]; Samko et al. [28] for details). The fractional derivative appearing in (2.7) must be understood as follows

$$
\frac{d^{\alpha}}{d x^{\alpha}} f(x)=\frac{1}{\Gamma(n-\alpha)} \int_{0}^{x}(x-s)^{n-\alpha-1} \frac{d^{n} f}{d s^{n}}(s) d s, \quad n-1<\alpha<n
$$

that is the Dzerbayshan-Caputo sense. We also deal with the Riemann-Liouville fractional derivative

$$
D_{x}^{\alpha} f=\frac{1}{\Gamma(n-\alpha)} \frac{d^{n}}{d x^{n}} \int_{0}^{x}(x-s)^{n-\alpha-1} f(s) d s, \quad n-1<\alpha<n
$$


and the fact that

$$
D_{x}^{\alpha} f=\frac{d^{\alpha}}{d x^{\alpha}} f-\left.\sum_{k=0}^{n-1} \frac{d^{k}}{d x^{k}} f\right|_{x=0^{+}} \frac{x^{k-\alpha}}{\Gamma(k-\alpha+1)}, \quad n-1<\alpha<n,
$$

see Gorenflo and Mainardi [11] and Kilbas et al. [13]. We refer to Kilbas et al. [13]; Samko et al. [28] for a close examination of the fractional derivatives (2.8) and (2.9).

\section{Mellin convolution of generalized Gamma densities}

In this section we introduce and study the Mellin convolution of generalized gamma densities. In the literature, it is well-known that generalized Gamma r.v. possess density law given by

$$
Q_{\mu}^{\gamma}(z)=\gamma \frac{z^{\gamma \mu-1}}{\Gamma(\mu)} \exp \left\{-z^{\gamma}\right\}, \quad z>0, \gamma>0, \mu>0 .
$$

Our discussion here concerns the function

$$
g_{\mu}^{\gamma}(x, t)=\operatorname{sign}(\gamma) \frac{1}{t} Q_{\mu}^{\gamma}\left(\frac{x}{t}\right)=|\gamma| \frac{x^{\gamma \mu-1}}{t^{\gamma \mu} \Gamma(\mu)} \exp \left\{-\frac{x^{\gamma}}{t^{\gamma}}\right\}, \quad x>0, t>0, \gamma \neq 0, \mu>0 .
$$

Let us introduce the convolution

$$
g_{\mu_{1}}^{\gamma_{1}} \star g_{\mu_{2}}^{\gamma_{2}}(x, t)=\int_{0}^{\infty} g_{\mu_{1}}^{\gamma_{1}}(x, s) g_{\mu_{2}}^{\gamma_{2}}(s, t) d s=\operatorname{sign}\left(\gamma_{1} \gamma_{2}\right) \frac{1}{t} \int_{0}^{\infty} Q_{\mu_{1}}^{\gamma_{1}}(x / s) Q_{\mu_{2}}^{\gamma_{2}}(s / t) \frac{d s}{s}
$$

for which we have (see formula (2.4))

$$
\mathscr{M}\left[g_{\mu_{1}}^{\gamma_{1}} \star g_{\mu_{2}}^{\gamma_{2}}(\cdot, t)\right](\eta)=\mathscr{M}\left[g_{\mu_{1}}^{\gamma_{1}}\left(\cdot, t^{1 / 2}\right)\right](\eta) \times \mathscr{M}\left[g_{\mu_{2}}^{\gamma_{2}}\left(\cdot, t^{1 / 2}\right)\right](\eta)
$$

as a straightforward calculation shows. We now introduce the generalized Gamma process (GGP in short). Roughly speaking, the function (3.1) can be viewed as the distribution of a GGP $\left\{G_{t}^{\gamma, \mu}, t>\right.$ $0\}$ in the sense that $\forall t$ the distribution of the r.v. $G_{t}^{\gamma, \mu}$ is the generalized Gamma distribution (3.1). Thus, we make some abuse of language by considering a process without its covariance structure. In the literature there are several non-equivalent definitions of the distribution on $\mathbb{R}_{+}^{n}$ of Gamma distributions, see e.g. Kotz et al. [16] for a comprehensive discussion. In Section 5 (Corollary 1) we will show that the distribution (3.1) satisfies the p.d.e.

$$
\frac{\partial}{\partial t} g_{\mu}^{\gamma}=\frac{d\left(t^{\gamma}\right)}{d t} \mathscr{G}_{\gamma, \mu} g_{\mu}^{\gamma}, \quad x>0, t>0
$$

where

$$
\mathscr{G}_{\gamma, \mu} f=\frac{1}{\gamma^{2}}\left(\frac{\partial}{\partial x} x^{2-\gamma} \frac{\partial}{\partial x}-(\gamma \mu-1) \frac{\partial}{\partial x} x^{1-\gamma}\right) f, \quad x>0, t>0
$$

and $\gamma \neq 0, f \in D\left(\mathscr{G}_{\gamma, \mu}\right)$. For $\gamma=1$, equation (3.1) becomes the distribution of a $2 \mu$-dimensional squared Bessel process $\left\{B E S S Q_{t / 2}^{(2 \mu)}, t>0\right\}$ and, for $\gamma=2$ we obtain the distribution of a $2 \mu$ dimensional Bessel process $\left\{B E S_{t / 2}^{(2 \mu)}, t>0\right\}$, both starting from zero. Some interesting distributions can be realized through Mellin convolution of distribution $g_{\mu}^{\gamma}$. Indeed, after some algebra we arrive at

$$
g_{\mu_{1}}^{\gamma} \star g_{\mu_{2}}^{-\gamma}(x, t)=\frac{\gamma}{B\left(\mu_{1}, \mu_{2}\right)} \frac{x^{\gamma \mu_{1}-1} t^{\gamma \mu_{2}}}{\left(t^{\gamma}+x^{\gamma}\right)^{\mu_{1}+\mu_{2}}}, \quad x>0, t>0, \gamma>0
$$


and

$$
g_{\mu_{1}}^{-\gamma} \star g_{\mu_{2}}^{\gamma}(x, t)=\frac{\gamma}{B\left(\mu_{1}, \mu_{2}\right)} \frac{x^{\gamma \mu_{2}-1} t^{\gamma \mu_{1}}}{\left(t^{\gamma}+x^{\gamma}\right)^{\mu_{1}+\mu_{2}}}, \quad x>0, t>0, \gamma>0
$$

where $B(\cdot, \cdot)$ is the Beta function (see e.g. Gradshteyn and Ryzhik [12, formula 8.384]). Moreover, in light of the Mellin convolution formula (2.4), the following holds true

$$
\mathscr{M}\left[g_{\mu_{1}}^{\gamma} \star g_{\mu_{2}}^{-\gamma}(\cdot, t)\right](\eta)=\mathscr{M}\left[g_{\mu_{2}}^{-\gamma} \star g_{\mu_{1}}^{\gamma}(\cdot, t)\right](\eta) \text {. }
$$

A further distribution arising from convolution can be presented. In particular, for $\gamma \neq 0$, we have

$$
g_{\mu_{1}}^{\gamma} \star g_{\mu_{2}}^{\gamma}(x, t)=\frac{2|\gamma|\left(x^{\gamma} / t^{\gamma}\right)^{\frac{\mu_{1}+\mu_{2}}{2}}}{x \Gamma\left(\mu_{1}\right) \Gamma\left(\mu_{2}\right)} K_{\mu_{2}-\mu_{1}}\left(2 \sqrt{\frac{x^{\gamma}}{t^{\gamma}}}\right), \quad x>0, t>0
$$

which proves to be very useful further on. The function $K_{v}$ appearing in (3.7) is the modified Bessel function of imaginary argument (see e.g [12, formula 8.432]). For the sake of completeness we have writen the following Mellin transforms:

$$
\mathscr{M}\left[g_{\mu}^{\gamma}(\cdot, t)\right](\eta)=\frac{\Gamma\left(\frac{\eta-1}{\gamma}+\mu\right)}{\Gamma(\mu)} t^{\eta-1}, \quad t>0, \Re\{\eta\}>1-\gamma \mu, \gamma \neq 0,
$$

and

$$
\mathscr{M}\left[g_{\mu}^{\gamma}(x, \cdot)\right](\eta)=\frac{\Gamma\left(\mu-\frac{\eta}{\gamma}\right)}{\Gamma(\mu)} x^{\eta-1}, \quad x>0, \Re\{\eta\}>\gamma \mu, \gamma \neq 0 .
$$

Formula (3.8) suggests that

$$
\mathscr{M}\left[g_{\mu_{1}}^{\gamma_{1}} \star g_{\mu_{2}}^{\gamma_{2}}(x, \cdot)\right](\eta)=\mathscr{M}\left[g_{\mu_{1}}^{\gamma_{1}}\left(x^{1 / 2}, \cdot\right)\right](\eta) \times \mathscr{M}\left[g_{\mu_{2}}^{\gamma_{2}}\left(x^{1 / 2}, \cdot\right)\right](\eta) .
$$

For the one-dimensional GGP we are able to define the inverse generalized Gamma process $\left\{E_{t}^{\gamma, \mu}\right.$, $t>0\}$ (IGGP in short) by means of the following relation

$$
\operatorname{Pr}\left\{E_{t}^{\gamma, \mu}<x\right\}=\operatorname{Pr}\left\{G_{x}^{\gamma, \mu}>t\right\} .
$$

The density law $e_{\mu}^{\gamma}=e_{\mu}^{\gamma}(x, t)$ of the IGGP can be carried out by observing that

$$
e_{\mu}^{\gamma}(x, t)=\operatorname{Pr}\left\{E_{t}^{\gamma, \mu} \in d x\right\} / d x=\int_{t}^{\infty} \frac{\partial}{\partial x} g_{\mu}^{\gamma}(s, x) d s, \quad x>0, t>0
$$

and, making use of the Mellin transform, we obtain

$$
\begin{aligned}
\mathscr{M}\left[e_{\mu}^{\gamma}(\cdot, t)\right](\eta) & =\int_{t}^{\infty} \mathscr{M}\left[\frac{\partial}{\partial x} g_{\mu}^{\gamma}(s, \cdot)\right](\eta) d s, \quad \Re\{\eta\}<1 \\
& =[\text { by (2.6) }]=-(\eta-1) \int_{t}^{\infty} \mathscr{M}\left[g_{\mu}^{\gamma}(s, \cdot)\right](\eta-1) d s \\
& =[\text { by (3.8) }]=-(\eta-1) \int_{t}^{\infty} \frac{\Gamma\left(\mu-\frac{\eta-1}{\gamma}\right)}{\Gamma(\mu)} s^{\eta-2} d s=\frac{\Gamma\left(\mu-\frac{\eta-1}{\gamma}\right)}{\Gamma(\mu)} t^{\eta-1}
\end{aligned}
$$


The derivative under the integral sign in (3.9) is allowed from the fact that $\Xi_{1}(s)=\frac{\partial}{\partial x} g_{\mu}^{\gamma}(s, x) \in$ $L^{1}\left(\mathbb{R}_{+}\right)$as a function of $s$. From (2.2) and the fact that

$$
H_{p, q}^{m, n}\left[x \mid \begin{array}{l}
\left(a_{i}, \alpha_{i}\right)_{i=1, \ldots, p} \\
\left(b_{j}, \beta_{j}\right)_{j=1, . ., q}
\end{array}\right]=c H_{p, q}^{m, n}\left[\begin{array}{l|l}
c & \begin{array}{l}
\left(a_{i}, c \alpha_{i}\right)_{i=1, \ldots, p} \\
\left(b_{j}, c \beta_{j}\right)_{j=1, . ., q}
\end{array}
\end{array}\right]
$$

for all $c>0$ (see Mathai and Saxena [20]), we have that

$$
e_{\mu}^{\gamma}(x, t)=\frac{\gamma}{x} H_{1,1}^{1,0}\left[\frac{t^{\gamma}}{x^{\gamma}} \mid \begin{array}{l}
(\mu, 0) \\
(\mu, 1)
\end{array}\right], \quad x>0, t>0, \gamma>0 .
$$

By observing that $\mathscr{M}\left[e_{\mu}^{\gamma}(\cdot, t)\right](1)=1$, we immediately verify that (3.11) integrates to unity. The density law $g_{\mu}^{\gamma}$ can be expressed in terms of $\mathrm{H}$ functions as well, therefore we have

$$
g_{\mu}^{\gamma}(x, t)=\frac{\gamma}{x} H_{1,1}^{1,0}\left[\frac{x^{\gamma}}{t^{\gamma}} \mid \begin{array}{c}
(\mu, 0) \\
(\mu, 1)
\end{array}\right], \quad x>0, t>0, \gamma>0 .
$$

In view of (3.11) and (3.12) we can argue that

$$
E_{t}^{\gamma, \mu} \stackrel{\text { law }}{=} G_{t}^{-\gamma, \mu} \stackrel{\text { law }}{=} 1 / G_{t}^{\gamma, \mu}, \quad t>0, \gamma>0, \mu>0
$$

and $e_{\mu}^{\gamma}(x, t)=g_{\mu}^{-\gamma}(x, t), \gamma>0, x>0, t>0$.

Remark 1. We notice that the inverse process $\left\{E_{t}^{1,1 / 2}, t>0\right\}$ can be written as

$$
E_{t}^{1,1 / 2}=\inf \{s ; B(s)=\sqrt{2 t}\}
$$

where $B$ is a standard Brownian motion. Thus, $E^{1,1 / 2}$ can be interpreted as the first-passage time of a standard Brownian motion through the level $\sqrt{2 t}$.

In what follows we will consider the Mellin convolution $e_{\bar{\mu}}^{\star n}(x, t)=e_{\mu_{1}} \star \ldots \star e_{\mu_{n}}(x, t)$ (see formulae (2.4) and (3.2)) where $\bar{\mu}=\left(\mu_{1}, \ldots, \mu_{n}\right), \mu_{j}>0, j=1,2, \ldots, n$ and, for the sake of simplicity, $e_{\mu}(x, t)=e_{\mu}^{1}(x, t)$. For the density law $e_{\bar{\mu}}^{\star n}(x, t), x>0, t>0$ we have

$$
\mathscr{M}\left[e_{\bar{\mu}}^{\star n}(\cdot, t)\right](\eta)=\prod_{j=1}^{n} \mathscr{M}\left[e_{\mu_{j}}\left(\cdot, t^{1 / n}\right)\right](\eta)=t^{\eta-1} \prod_{j=1}^{n} \frac{\Gamma\left(\mu_{j}+1-\eta\right)}{\Gamma\left(\mu_{j}\right)}
$$

with $\Re\{\eta\}<1$. Furthermore, for the Mellin convolution $g_{\bar{\mu}}^{\gamma, \star n}(x, t)=g_{\mu_{1}}^{\gamma} \star, \ldots, \star g_{\mu_{n}}^{\gamma}(x, t)$ we have

$$
\mathscr{M}\left[g_{\bar{\mu}}^{\gamma, \star n}(\cdot, t)\right](\eta)=\prod_{j=1}^{n} \mathscr{M}\left[g_{\mu_{j}}^{\gamma}\left(\cdot, t^{1 / n}\right)\right](\eta)=t^{\eta-1} \prod_{j=1}^{n} \frac{\Gamma\left(\frac{\eta-1}{\gamma}+\mu_{j}\right)}{\Gamma\left(\mu_{j}\right)}
$$

with $\Re\{\eta\}>1-\min _{j}\left\{\mu_{j}\right\}$.

Lemma 1. The functions $g_{\mu}^{\gamma}$ and $e_{\mu}^{\gamma}$ are commutative under $\star$-convolution.

Proof. Consider the Mellin convolution (3.13). Let $e_{\mu_{j}}$ be the distribution of the process $X^{\sigma_{j}}$, then formula (3.13) means that

$$
E\left\{X^{\sigma_{1}}\left(X^{\sigma_{2}}\left(\ldots X^{\sigma_{n}}(t) \ldots\right)\right)\right\}^{\eta-1}=E\left\{X^{\sigma_{1}}\left(t^{1 / n}\right) X^{\sigma_{2}}\left(t^{1 / n}\right) \cdots X^{\sigma_{n}}\left(t^{1 / n}\right)\right\}^{\eta-1}
$$

for all possible permutations of $\left\{\sigma_{j}\right\}, j=1,2, \ldots, n$. The same result can be shown for $e_{\mu_{j}}^{\gamma}$. Suppose now that the process $X^{\sigma_{j}}$ possesses distribution $g_{\mu_{j}}^{\gamma}$, from (3.14) we obtain the claimed result. 


\section{Stable subordinators}

The $v$-stable subordinators $\left\{\tilde{\tau}_{t}^{(v)}, t>0\right\}, v \in(0,1)$, are defined as non-decreasing, (totally) positively skewed, Lévy processes with Laplace transform

$$
E \exp \left\{-\lambda \tilde{\tau}_{t}^{(v)}\right\}=\exp \left\{-t \lambda^{v}\right\}, \quad t>0, \quad \lambda>0
$$

and characteristic function

$$
E \exp \left\{i \xi \tilde{\tau}_{t}^{(v)}\right\}=\exp \left\{-t \Psi_{v}(\xi)\right\}, \quad \xi \in \mathbb{R}
$$

where

$$
\Psi_{v}(\xi)=\int_{0}^{\infty}\left(1-e^{-i \xi u}\right) \frac{v}{\Gamma(1-v)} \frac{d u}{u^{v+1}}
$$

(see Bertoin [4]; Zolotarev [32]). After some algebra we get

$$
\Psi_{v}(\xi)=\sigma|\xi|^{v}\left(1-i \operatorname{sgn}(\xi) \tan \left(\frac{\pi v}{2}\right)\right)=|\xi|^{v} \exp \left\{-i \frac{\pi v}{2} \frac{\xi}{|\xi|}\right\} .
$$

For the density law of the $v$-stable subordinator $\left\{\tilde{\tau}_{t}^{(v)}, t>0\right\}$, say $h_{v}=h_{v}(x, t), x>0, t>0$ we have the $t$-Mellin transforms

$$
\mathscr{M}\left[\hat{h}_{v}(\xi, \cdot)\right](\eta)=|\xi|^{-\eta v} \exp \left\{i \frac{\pi \eta v}{2} \frac{\xi}{|\xi|}\right\} \Gamma(\eta)
$$

and

$$
\mathscr{M}\left[\tilde{h}_{v}(\lambda, \cdot)\right](\eta)=\lambda^{-\eta v} \Gamma(\eta)
$$

where $\hat{h}_{v}(\xi, t)=\mathscr{F}\left[h_{v}(\cdot, t)\right](\xi)$ is the Fourier transform appearing in 4.2) and $\tilde{h}_{v}(\lambda, t)=$ $\mathscr{L}\left[h_{v}(\cdot, t)\right](\lambda)$ is the Laplace transform (4.1). By inverting (4.3) we obtain the Mellin transform with respect to $t$ of the density $h_{v}$ which reads

$$
\begin{aligned}
\mathscr{M}\left[h_{v}(x, \cdot)\right](\eta) & =\frac{1}{2 \pi} \int_{\mathbb{R}} e^{-i \xi x} \mathscr{M}[\hat{h}(\xi, \cdot)](\eta) d \xi \\
& =\frac{\Gamma(\eta) \Gamma(1-\eta v)}{2 \pi}\left\{\frac{e^{i \frac{\pi \eta v}{2}}}{(i x)^{1-\eta v}}+\frac{e^{-i \frac{\pi \eta v}{2}}}{(-i x)^{1-\eta v}}\right\} \\
& =\frac{\Gamma(\eta) \Gamma(1-\eta v)}{2 \pi x^{1-\eta v}}\left\{\exp \left\{-i \frac{\pi}{2}+i \pi \eta v\right\}+\exp \left\{i \frac{\pi}{2}-i \pi \eta v\right\}\right\} \\
& =\frac{\Gamma(\eta) \Gamma(1-\eta v)}{\pi x^{1-\eta v}} \sin \pi \eta v=\frac{\Gamma(\eta)}{\Gamma(\eta v)} x^{\eta v-1}, \quad x>0, v \in(0,1)
\end{aligned}
$$

where $\Re\{\eta v\} \in(0,1)$. Formula (4.5) can be also obtained by inverting (4.4). We are also able to evaluate the Mellin transform with respect to $x$ of the density law $h_{v}$. From (4.3) and the fact that

$$
\int_{0}^{\infty} x^{\eta-1} e^{-i \xi x} d x=\frac{\Gamma(\eta)}{(i \xi)^{\eta}}, \quad \text { where } \quad( \pm i \xi)^{v}=|\xi|^{v} \exp \left\{ \pm i \frac{v \pi}{2} \frac{\xi}{|\xi|}\right\}, \quad v \in(0,1)
$$

we obtain

$$
\mathscr{M}\left[h_{v}(\cdot, t)\right](\eta)=\frac{\Gamma(\eta)}{2 \pi} \int_{\mathbb{R}}|\xi|^{-\eta} \exp \left\{-i \frac{\pi \eta}{2} \frac{\xi}{|\xi|}-t \Psi_{v}(\xi)\right\} d \xi
$$




$$
\begin{aligned}
& =\frac{\Gamma(\eta)}{2 \pi}\left\{e^{-i \frac{\pi \eta}{2}} \int_{0}^{\infty} \xi^{-\eta} e^{-t \Phi_{v}(\xi)} d \xi+e^{i \frac{\pi \eta}{2}} \int_{0}^{\infty} \xi^{-\eta} e^{-t \Phi_{v}(-\xi)} d \xi\right\} \\
& =\frac{\Gamma(\eta)}{2 \pi v} \Gamma\left(\frac{1-\eta}{v}\right) t^{\frac{\eta-1}{v}}\left\{e^{i \pi(1-\eta)}+e^{-i \pi(1-\eta)}\right\} \\
& =\Gamma\left(\frac{1-\eta}{v}\right) \frac{t^{\frac{\eta-1}{v}}}{v \Gamma(1-\eta)}, \quad \Re\{\eta\} \in(0,1), t>0 .
\end{aligned}
$$

The inversion of Fourier and Laplace transforms by making use of Mellin transform has been also treated by Schneider and Wyss [29].

We investigate the relationship between stable subordinators and their inverse processes. For a $v$-stable subordinator $\left\{\tilde{\tau}_{t}^{(v)}, t>0\right\}$ and an inverse process $\left\{L_{t}^{(v)}, t>0\right\}$ (ISP in short) such that

$$
\operatorname{Pr}\left\{L_{t}^{(v)}<x\right\}=\operatorname{Pr}\left\{\tilde{\tau}_{x}^{(v)}>t\right\}
$$

we have the following relationship between density laws

$$
l_{v}(x, t)=\operatorname{Pr}\left\{L_{t}^{(v)} \in d x\right\} / d x=\int_{t}^{\infty} \frac{\partial}{\partial x} h_{v}(s, x) d s, \quad x>0, t>0 .
$$

We observe that $\frac{\partial}{\partial x} h_{v}(s, x)$ exists and there exists $\zeta(s) \in L^{1}\left(\mathbb{R}_{+}\right)$such that $\Xi_{2}(s)=\frac{\partial}{\partial x} h_{v}(s, x)=$ const $\cdot D_{s}^{v} h_{v}(s, x) \leq \zeta(s)$. The function $h_{v}$ is the distribution of a totally skewed stable process, thus $h_{v}(\mathbf{x}), \mathbf{x} \in \mathbb{R}_{+}^{n}$ belongs to the space of functions in $D\left((-\triangle)^{v / 2}\right)$, see Samko et al. [28]. Thus, the integral in (4.8) converges. The density law (4.8) can be written in terms of Fox functions by observing that

$$
\begin{aligned}
\mathscr{M}\left[l_{v}(\cdot, t)\right](\eta) & =\int_{t}^{\infty} \mathscr{M}\left[\frac{\partial}{\partial x} h_{v}(s, \cdot)\right](\eta) d s \\
& =[\text { by (2.6)] }]=-(\eta-1) \int_{t}^{\infty} \mathscr{M}\left[h_{v}(s, \cdot)\right](\eta-1) d s \\
& =[\text { by } 4.5]]=-\int_{t}^{\infty} \frac{\Gamma(\eta)}{\Gamma(\eta v-v)} s^{\eta v-v-1} d s \\
& =\frac{\Gamma(\eta)}{\Gamma(\eta v-v+1)} t^{v(\eta-1)}, \quad \Re\{\eta\}<1 / v, t>0 .
\end{aligned}
$$

Thus, by direct inspection of 2.2 , we recognize that

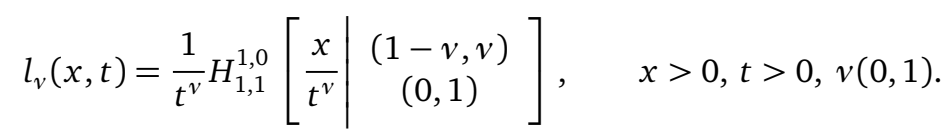

Density (4.10) integrates to unity, indeed $\mathscr{M}\left[l_{v}(\cdot, t)\right](1)=1$. The $t$-Laplace transform

$$
\mathscr{L}\left[l_{v}(x, \cdot)\right](\lambda)=\lambda^{v-1} \exp \left\{-x \lambda^{v}\right\}, \quad \lambda>0, v \in(0,1)
$$

comes directly from the fact that

$$
\int_{0}^{\infty} e^{-\lambda t} \mathscr{M}\left[l_{v}(\cdot, t)\right](\eta) d t=\frac{\Gamma(\eta)}{\lambda^{\eta v-v+1}}=\int_{0}^{\infty} x^{\eta-1} \mathscr{L}\left[l_{v}(x, \cdot)\right](\lambda) d x .
$$


From (4.11) we retrieve the well-known fact that $\mathscr{L}\left[l_{v}(\cdot, t)\right](\lambda)=E_{v}\left(-\lambda t^{v}\right)$ (see also Bondesson et al. [5]) where $E_{\beta}$ is the Mittag-Leffler function which can be also written as

$$
E_{v}\left(-\lambda t^{v}\right)=\frac{1}{\pi} \int_{0}^{\infty} \exp \left\{-\lambda^{1 / v} t x\right\} \frac{x^{v-1} \sin \pi v}{1+2 x^{v} \cos \pi v+x^{2 v}} d x, \quad t>0, \lambda>0 .
$$

The distribution $l_{v}$ satisfies the fractional equation $\frac{\partial^{v}}{\partial t^{v}} l_{v}=-\frac{\partial}{\partial x} l_{v}, x>0, t>0$ subject to $l_{v}(x, 0)=\delta(x)$ where the fractional derivative must be understood in the Dzerbayshan-Caputo sense (formula (2.8)). The governing equation of $l_{v}$ can be also presented by considering the Riemann-Liouville derivative (2.9) and the relation (2.10) (see e.g. Baeumer and Meerschaert [1]; Meerschaert and Scheffler [21]; Baeumer et al. [2]). It is well-known that the ratio involving two independent stable subordinator $\left\{{ }_{1} \tilde{\tau}_{t}^{(v)}, t>0\right\}$ and $\left\{{ }_{2} \tilde{\tau}_{t}^{(v)}, t>0\right\}$ has a distribution, $\forall t$, given by

$$
r(w)=\operatorname{Pr}\left\{{ }_{1} \tilde{\tau}_{t}^{(v)} /{ }_{2} \tilde{\tau}_{t}^{(v)} \in d w\right\} / d w=\frac{1}{\pi} \frac{w^{v-1} \sin \pi v}{1+2 w^{v} \cos \pi v+w^{2 v}}, \quad w>0, t>0 .
$$

Here we study the ratio of two independent inverse stable processes $\left\{{ }_{1} L_{t}^{(v)}, t>0\right\}$ and $\left\{{ }_{2} L_{t}^{(v)}, t>\right.$ $0\}$ by evaluating its Mellin transform as follows

$$
E\left\{{ }_{1} L_{t}^{(v)} /{ }_{2} L_{t}^{(v)}\right\}^{\eta-1}=\mathscr{M}\left[l_{v}(\cdot, t)\right](\eta) \times \mathscr{M}\left[l_{v}(\cdot, t)\right](2-\eta)=\frac{1}{v} \frac{\sin v \pi-\eta v \pi}{\sin \eta \pi}
$$

with $\Re\{\eta\} \in(0,1)$. By inverting (4.14) we obtain

$$
k(x)=\frac{1}{v \pi} \frac{\sin v \pi}{1+2 x \cos v \pi+x^{2}}=\frac{1}{2 \pi i} \int_{\theta-i \infty}^{\theta+i \infty} \frac{\sin v \pi-\eta v \pi}{\sin \eta \pi} x^{-\eta} d \eta
$$

for some real $\theta \in(0,1)$. From (4.13) and (4.15) we can argue that

$$
\left({ }_{1} \tilde{\tau}_{t}^{(v)} /{ }_{2} \tilde{\tau}_{t}^{(v)}\right)^{v} \stackrel{\text { law }}{=}{ }_{1} L_{t}^{(v)} /{ }_{2} L_{t}^{(v)}, \quad \forall t>0 .
$$

We notice that the equivalence in law (4.16) is independent of $t$ as the formulae (4.13) and (4.15) entail. The distribution $h_{v} \circ l_{v}(x, t)$ of the process $\left\{\tilde{\tau}_{L_{t}^{(v)}}^{(v)}, t>0\right\}$ has Mellin transform (by making use of the formulae (4.7) and (4.9)) given by

$$
\mathscr{M}\left[h_{v} \circ l_{v}(\cdot, t)\right](\eta)=\mathscr{M}\left[h_{v}(\cdot, 1)\right](\eta) \times \mathscr{M}\left[l_{v}(\cdot, t)\right]\left(\frac{\eta-1}{v}+1\right)=\frac{1}{v} \frac{\sin \pi \eta}{\sin \pi \frac{1-\eta}{v}} t^{\eta-1}, \quad t>0
$$

with $\Re\{\eta\} \in(0,1)$. Thus, we can infer that

$$
\tilde{\tau}_{L_{t}^{(v)}}^{(v)} \stackrel{\text { law }}{=} t \times{ }_{1} \tilde{\tau}_{t}^{(v)} /{ }_{2} \tilde{\tau}_{t}^{(v)} \quad t>0
$$

and $h_{v} \circ l_{v}(x, t)=t^{-1} r(x / t)$ where $r(w)$ is that in (4.13). For the process $\left\{L_{\tilde{\tau}_{t}^{(v)}}^{(v)}, t>0\right\}$ with distribution $l_{v} \circ h_{v}(x, t)$ we obtain (from (4.9) and 4.7))

$$
\mathscr{M}\left[l_{v} \circ h_{v}(\cdot, t)\right](\eta)=\mathscr{M}\left[l_{v}(\cdot, 1)\right](\eta) \times \mathscr{M}\left[h_{v}(\cdot, t)\right](\eta v-v+1)=\frac{1}{v} \frac{\sin \pi v-\pi \eta v}{\sin \pi \eta} t^{\eta-1}
$$

with $\Re\{\eta\} \in(0,1)$ and thus

$$
L_{\tau_{t}^{(v)}}^{(v)} \stackrel{\text { law }}{=} t \times{ }_{1} L_{t}^{(v)} /{ }_{2} L_{t}^{(v)}, \quad t>0 .
$$

We have that $l_{v} \circ h_{v}(x, t)=t^{-1} k(x / t)$ where $k(x)$ is that in 4.15). 


\section{Main results}

In this section we consider compositions of processes whose governing equations are (generalized) fractional diffusion equations. When we consider compositions involving Markov processes and stable subordinators we still have Markov processes. Here we study Markov processes with random time which is the inverse of a stable subordinator. Such a process does not belong to the family of stable subordinators (see (4.12) ) and the resultant composition is not, in general, a Markov process. This somehow explains the effect of the fractional derivative appearing in the governing equation, see Mainardi et al. [19]. Hereafter, we exploit the Mellin convolution of generalized Gamma densities in order to write explicitly the solutions to fractional diffusion equations. We first present a new representation of the density law $h_{v}$ by means of the convolution $e_{\bar{\mu}}^{\star n}$ introduced in Section 3. To do this we also introduce the time-stretching function $\varphi_{m}(s)=(s / m)^{m}, m \geq 1$, $s \in(0, \infty)$.

Lemma 2. The Mellin convolution $e_{\bar{\mu}}^{\star n}\left(x, \varphi_{n+1}(t)\right)$ where $\mu_{j}=j v$, for $j=1,2, \ldots, n$ is the density law of a $v$-stable subordinator $\left\{\tilde{\tau}_{t}^{(v)}, t>0\right\}$ with $v=1 /(n+1), n \in \mathbb{N}$. Thus, we have

$$
h_{v}(x, t)=e_{\bar{\mu}}^{\star n}\left(x, \varphi_{n+1}(t)\right), \quad x>0, t>0, v=1 /(n+1), n \in \mathbb{N} .
$$

Proof. From (3.13) we have that

$$
\mathscr{M}\left[e_{\bar{\mu}}^{\star n}\left(\cdot, \varphi_{n+1}(t)\right)\right](\eta)=\frac{\prod_{j=1}^{n} \Gamma\left(1-\eta+\mu_{j}\right)}{\prod_{j=1}^{n} \Gamma\left(\mu_{j}\right)}\left(\varphi_{n+1}(t)\right)^{\eta-1} .
$$

From Gradshteyn and Ryzhik [12, formula 8.335.3] we deduce that

$$
\prod_{k=1}^{n} \Gamma\left(\frac{k}{n+1}\right)=\frac{(2 \pi)^{\frac{n}{2}}}{\sqrt{n+1}}, \quad n \in \mathbb{N}
$$

and formula (5.1) reduces to

$$
\mathscr{M}\left[e_{\bar{\mu}}^{\star n}\left(\cdot, \varphi_{n+1}(t)\right)\right](\eta)=\frac{\prod_{j=1}^{n} \Gamma\left(1-\eta+\mu_{j}\right)}{(2 \pi)^{n / 2} \sqrt{v}}\left(\varphi_{n+1}(t)\right)^{\eta-1} .
$$

Furthermore, by making use of the (product theorem) relation

$$
\Gamma(n x)=(2 \pi)^{\frac{1-n}{2}} n^{n x-1 / 2} \prod_{k=0}^{n-1} \Gamma\left(x+\frac{k}{n}\right)
$$

(see Gradshteyn and Ryzhik [12, formula 3.335]) formula (5.3) becomes

$$
\mathscr{M}\left[e_{\bar{\mu}}^{\star n}\left(\cdot, \varphi_{n}(t)\right)\right](\eta)=\frac{\Gamma\left(\frac{1-\eta}{v}\right)(2 \pi)^{n / 2}(n+1)^{\eta / v-n}}{\Gamma(1-\eta)(2 \pi)^{n / 2}}\left(\varphi_{n+1}(t)\right)^{\eta-1}=\frac{\Gamma\left(\frac{1-\eta}{v}\right)}{v \Gamma(1-\eta)} t^{\frac{\eta-1}{v}}
$$

(with $\Re\{\eta\} \in(0,1)$ ) which coincides with (4.7). The claimed result is obtained. 
In light of the last result we are able to write explicitly the density law of a stable subordinator. For $v=1 / 2$, Lemma 2 says that

$$
h_{1 / 2}(x, t)=e_{\bar{\mu}}^{\star 1}\left(x, \varphi_{2}(t)\right)=e_{1 / 2}\left(x,(t / 2)^{2}\right)=\frac{x^{-1 / 2-1} e^{-\frac{t^{2}}{4 x}}}{t^{-1} \sqrt{4} \Gamma\left(\frac{1}{2}\right)}, \quad x>0, t>0
$$

which is the well-known density law of the $1 / 2$-stable subordinator or the first-passage time of a standard Brownian motion trough the level $t / \sqrt{2}$. For $v=1 / 3$, from (3.7), we obtain

$$
h_{1 / 3}(x, t)=e_{\bar{\mu}}^{\star 2}\left(x, \varphi_{3}(t)\right)=e_{1 / 3} \star e_{2 / 3}\left(x,(t / 3)^{3}\right)=\frac{1}{3 \pi} \frac{t^{3 / 2}}{x^{3 / 2}} K_{\frac{1}{3}}\left(\frac{2}{3^{3 / 2}} \frac{t^{3 / 2}}{\sqrt{x}}\right), \quad x>0, t>0 .
$$

For $v=1 / 4$, by (3.7) (and the commutativity under $\star$, see Lemma 1), we have

$$
h_{1 / 4}(x, t)=e_{\bar{\mu}}^{\star 3}\left(x, \varphi_{4}(t)\right)=e_{1 / 4} \star e_{2 / 4} \star e_{3 / 4}\left(x,(t / 4)^{4}\right)=e_{1 / 2} \star\left(e_{1 / 4} \star e_{3 / 4}\right)\left(x,(t / 4)^{4}\right)
$$

where $K_{1 / 2}(z)=\sqrt{\pi / 2 z} \exp \{-z\}$ (see [12, formula 8.469]). We notice that

$$
\mathscr{M}\left[e_{\bar{\mu}}^{\star 3}\left(\cdot, \varphi_{4}(t)\right)\right](\eta)=\mathscr{M}\left[h_{1 / 2} \circ h_{1 / 2}(\cdot, t)\right](\eta)
$$

which is in line with the well-known fact that

$$
E \exp \left\{-\lambda_{1} \tilde{\tau}_{2}^{\left(v_{1}\right)} \tilde{\tau}_{t}^{\left(v_{2}\right)}\right\}=E \exp \left\{-\lambda^{v_{1}}{ }_{2} \tilde{\tau}_{t}^{\left(v_{2}\right)}\right\}=\exp \left\{-t \lambda^{v_{1} v_{2}}\right\}
$$

$0<v_{i}<1, i=1,2$. For $v=1 / 5$, by exploiting twice (3.7) (and the commutativity under $\star$ ), we can write

$$
\begin{aligned}
h_{1 / 5}(x, t)=e_{\bar{\mu}}^{\star 4}\left(x,(t / 5)^{5}\right) & =\left(e_{1 / 5} \star e_{2 / 5}\right) \star\left(e_{3 / 5} \star e_{4 / 5}\right)\left(x,(t / 5)^{5}\right) \\
& =\frac{t^{7 / 2}}{5^{3} \pi^{2} x^{3 / 10+1}} \int_{0}^{\infty} s^{-2 / 5-1} K_{\frac{1}{5}}\left(2 \sqrt{\frac{s}{x}}\right) K_{\frac{1}{5}}\left(\frac{2}{5^{5 / 2}} \frac{t^{5 / 2}}{\sqrt{s}}\right) d s
\end{aligned}
$$

or equivalently

$$
\begin{aligned}
h_{1 / 5}(x, t)=e_{\bar{\mu}}^{\star 4}\left(x,(t / 5)^{5}\right) & =\left(e_{1 / 5} \star e_{3 / 5}\right) \star\left(e_{2 / 5} \star e_{4 / 5}\right)\left(x,(t / 5)^{5}\right) \\
& =\frac{t^{3}}{5^{5 / 2} \pi^{2} x^{2 / 5+1}} \int_{0}^{\infty} s^{-1 / 5-1} K_{\frac{2}{5}}\left(2 \sqrt{\frac{s}{x}}\right) K_{\frac{2}{5}}\left(\frac{2}{5^{5 / 2}} \frac{t^{5 / 2}}{\sqrt{s}}\right) d s .
\end{aligned}
$$

For $v=1 /(2 n+1), n \in \mathbb{N}$, by using repeatedly (3.7) we arrive at

$$
h_{v}(x, t)=\frac{x^{v / 2} t^{1 / v-3 / 2}}{v^{2-1 / v} \pi^{1 / 2 v-1 / 2}} \mathscr{K}_{v}^{\circ n}\left(x,(v t)^{1 / v}\right), \quad x>0, t>0
$$

where

$$
\mathscr{K}_{v}^{\circ n}(x, t)=\int_{0}^{\infty} \ldots \int_{0}^{\infty} \mathscr{K}_{v}\left(x, s_{1}\right) \ldots \mathscr{K}_{v}\left(s_{n-1}, t\right) d s_{1} \ldots d s_{n-1}
$$

is the integral (2.5) (as the symbol "o $n$ " denote) where $n$ functions are involved and $\mathscr{K}_{v}(x, t)=$ $x^{-2 v-1} K_{v}(2 \sqrt{t / x}), x>0, t>0$. We state a similar result for the density law $l_{v}$ and the convolution $g_{\bar{\mu}}^{\gamma, \star n}$ (see Section 3). Let us consider the time-stretching function $\psi_{m}(s)=m s^{1 / m}$, $s \in(0, \infty), m \in \mathbb{N},\left(\psi=\varphi^{-1}\right.$ where $\varphi$ has been introduced in the previous Lemma). 
Lemma 3. The Mellin convolution $g_{\bar{\mu}}^{(n+1), \star n}\left(x, \psi_{n+1}(t)\right)$ where $\mu_{j}=j v, j=1,2, \ldots, n$ and $v=$ $1 /(n+1), n \in \mathbb{N}$, is the density law of a $v$-inverse process $\left\{L_{t}^{(v)}, t>0\right\}$. Thus, we have

$$
l_{v}(x, t)=g_{\bar{\mu}}^{(n+1), \star n}\left(x, \psi_{n+1}(t)\right), \quad x>0, t>0, v=1 /(n+1), n \in \mathbb{N} .
$$

Proof. The proof can be carried out as the proof of Lemma 2 .

We obtain that $l_{1 / 2}(x, t)=g_{1 / 2}^{2}\left(x, 2 t^{1 / 2}\right)=e^{-\frac{x^{2}}{4 t}} / \sqrt{\pi t}, x>0, t>0$. Moreover, by making use of (3.7) and (5.2), we have that

$$
l_{1 / 3}(x, t)=g_{2 / 3}^{3} \star g_{1 / 3}^{3}\left(x, 3 t^{1 / 3}\right)=\frac{1}{\pi} \sqrt{\frac{x}{t}} K_{\frac{1}{3}}\left(\frac{2}{3^{3 / 2}} \frac{x^{3 / 2}}{\sqrt{t}}\right), \quad x>0, t>0
$$

and $l_{1 / 4}(x, t)=g_{3 / 4}^{4} \star g_{2 / 4}^{4} \star g_{1 / 4}^{4}\left(x, 4 t^{1 / 4}\right)$ follows (thank to the commutativity under $\star$ ) from

$$
g_{3 / 4}^{4} \star g_{2 / 4}^{4} \star g_{1 / 4}^{4}(x, t)=g_{1 / 2}^{4} \star\left(g_{3 / 4}^{4} \star g_{1 / 4}^{4}\right)(x, t)=\frac{2^{3 / 2}}{\pi} \frac{x}{t} \int_{0}^{\infty} \exp \left\{-(s x)^{4}-\frac{2}{(s t)^{2}}\right\} d s
$$

where $g_{3 / 4}^{4} \star g_{1 / 4}^{4}(x, t)$ is given by (3.7) and $K_{1 / 2}(z)=\sqrt{\pi / 2 z} \exp \{-z\}$ (see [12, formula 8.469]). In a more general setting, by making use of (3.7) we can write down

$$
g_{\bar{\mu}}^{1 / v, \star(1 / v-1)}(x, t)=\frac{1}{v^{1 / 2 v}}\left(\frac{x}{\pi^{2} t^{3}}\right)^{\frac{1-v}{4 v}} \mathscr{Q}_{\frac{1-v}{2}}^{\circ n}(x, t), \quad v=1 /(2 n+1), n \in \mathbb{N}
$$

where the symbol "o $n$ " stands for the integral (2.5) where $n$ functions $\mathscr{Q}_{\frac{1-v}{2}}$ are involved and

$$
\mathscr{Q}_{\frac{1-v}{2}}(x, t)=K_{\frac{1-v}{2}}\left(2 \sqrt{(x / t)^{1 / v}}\right), \quad x>0, t>0 .
$$

Now, we present the main result of this paper concerning the explicit solutions to (generalized) fractional diffusion equations. We study a generalized problem which leads to fractional diffusion equations involving the adjoint operators of both Bessel and squared Bessel processes. Let us introduce the distribution $\tilde{u}_{v}^{\gamma, \mu}=\tilde{g}_{\mu}^{\gamma} \circ l_{v}$ where $\tilde{g}_{\mu}^{\gamma}(x, t)=g_{\mu}^{\gamma}\left(x, t^{1 / \gamma}\right)$ and the Mellin transform of $\tilde{u}_{v}^{\gamma, \mu}$ which reads

$$
\mathscr{M}\left[\tilde{u}_{v}^{\gamma, \mu}(\cdot, t)\right](\eta)=\frac{\Gamma\left(\frac{\eta-1}{\gamma}+\mu\right) \Gamma\left(\frac{\eta-1}{\gamma}+1\right)}{\Gamma(\mu) \Gamma\left(\frac{\eta-1}{\gamma} v+1\right)} t^{\frac{\eta-1}{\gamma} v}, \quad 1-\gamma \mu<\Re\{\eta\}<1+\gamma / v-\gamma .
$$

We state the following result.

Theorem 1. Let the previous setting prevail. For $v=1 /(2 n+1), n \in \mathbb{N} \cup\{0\}$, the solutions to

$$
D_{t}^{v} \tilde{u}_{v}^{\gamma, \mu}=\mathscr{G}_{\gamma, \mu} \tilde{u}_{v}^{\gamma, \mu}, \quad x>0, t>0
$$

can be represented in terms of generalized Gamma convolution as

$$
\tilde{u}_{v}^{\gamma, \mu}(x, t)=\gamma \frac{x^{2 \mu-1} v^{\frac{1-3 v}{4 v}}}{\left(\pi^{2} t^{3 v}\right)^{\frac{1-v}{4 v}}} \int_{0}^{\infty} s^{\frac{1}{4 v}-\frac{1}{4}-\mu} e^{-x^{\gamma} / s} v_{v}(s, t) d s, \quad x \geq 0, t>0
$$


where $\mathscr{G}_{\gamma, \mu}$ is the operator appearing in (3.4),

$$
v_{v}(s, t)=\int_{0}^{\infty} \ldots \int_{0}^{\infty} \mathscr{Q}_{\frac{1-v}{2}}\left(s, s_{1}\right) \ldots \mathscr{Q}_{\frac{1-v}{2}}\left(s_{n-1}, t^{v} / v\right) d s_{1} \ldots d s_{n-1}
$$

and $\mathscr{Q}_{\frac{1-v}{2}}$ is that in (5.9). Moreover, for $v \in(0,1]$, we have

$$
\tilde{u}_{v}^{\gamma, \mu}(x, t)=\frac{\gamma}{x t^{v / \gamma}} H_{2,2}^{2,0}\left[\frac{x^{\gamma}}{t^{v}} \mid \begin{array}{ll}
(1, v) ; & (\mu, 0) \\
(1,1) ; & (\mu, 1)
\end{array}\right]
$$

in terms of $H$ Fox functions.

Proof. By exploiting the property (2.6) of the Mellin transform and the fact that

$$
\int_{0}^{\infty} x^{\eta-1} x^{\theta} f(x) d x=\mathscr{M}[f(\cdot)](\eta+\theta),
$$

for the operator (3.4) we have that

$$
\begin{aligned}
& \mathscr{M}\left[\mathscr{G}_{\gamma, \mu} \tilde{u}_{v}^{\gamma, \mu}(\cdot, t)\right](\eta) \\
& =-\frac{1}{\gamma^{2}}(\eta-1) \mathscr{M}\left[\frac{\partial}{\partial x} \tilde{u}_{v}^{\gamma, \mu}(\cdot, t)\right](\eta-\gamma+1)+\frac{1}{\gamma^{2}}(\gamma \mu-1)(\eta-1) \mathscr{M}\left[\tilde{u}_{v}^{\gamma, \mu}(\cdot, t)\right](\eta-\gamma) \\
& =\frac{1}{\gamma^{2}}(\eta-1)(\eta-\gamma) \mathscr{M}\left[\tilde{u}_{v}^{\gamma, \mu}(\cdot, t)\right](\eta-\gamma)+\frac{1}{\gamma^{2}}(\gamma \mu-1)(\eta-1) \mathscr{M}\left[\tilde{u}_{v}^{\gamma, \mu}(\cdot, t)\right](\eta-\gamma) \\
& =\frac{1}{\gamma^{2}}(\eta-1)(\eta-1+\gamma \mu-\gamma) \mathscr{M}\left[\tilde{u}_{v}^{\gamma, \mu}(\cdot, t)\right](\eta-\gamma)
\end{aligned}
$$

where $\mathscr{M}\left[\tilde{u}_{v}^{\gamma, \mu}(\cdot, t)\right](\eta)$ is that in $(5.10)$. We obtain

$$
\begin{aligned}
\mathscr{M}\left[\mathscr{G}_{\gamma, \mu} \tilde{u}_{v}^{\gamma, \mu}(\cdot, t)\right](\eta) & =\frac{1}{\gamma^{2}}(\eta-1)(\eta-1+\gamma \mu-\gamma) \frac{\Gamma\left(\frac{\eta-\gamma-1}{\gamma}+\mu\right) \Gamma\left(\frac{\eta-\gamma-1}{\gamma}+1\right)}{\Gamma(\mu) \Gamma\left(\frac{\eta-\gamma-1}{\gamma} v+1\right)} t^{\frac{\eta-\gamma-1}{\gamma} v} \\
& =\frac{1}{\gamma}(\eta-1) \frac{\Gamma\left(\frac{\eta-1}{\gamma}+\mu\right) \Gamma\left(\frac{\eta-1}{\gamma}\right)}{\Gamma(\mu) \Gamma\left(\frac{\eta-1}{\gamma} v-v+1\right)} t^{\frac{\eta-1}{\gamma} v-v} \\
& =\frac{\Gamma\left(\frac{\eta-1}{\gamma}+\mu\right) \Gamma\left(\frac{\eta-1}{\gamma}+1\right)}{\Gamma(\mu) \Gamma\left(\frac{\eta-1}{\gamma} v-v+1\right)} t^{\frac{\eta-1}{r} v-v}=D_{t}^{v} \mathscr{M}\left[\tilde{u}_{v}^{\gamma, \mu}(\cdot, t)\right](\eta)
\end{aligned}
$$

and $\tilde{u}_{v}^{\gamma, \mu}(x, t)$ solves (5.11) for $v \in(0,1)$. In view of Lemma 3 we can write

$$
\tilde{u}_{v}^{\gamma, \mu}(x, t)=\int_{0}^{\infty} \tilde{g}_{\mu}^{\gamma}(x, s) g_{\bar{\mu}}^{1 / v, \star(1 / v-1)}\left(s, \psi_{1 / v}(t)\right) d s
$$

and by means of (5.8) result (5.12) appears. Formula (5.13) follows directly from (2.2) by considering formula (3.10) and the fact that

$$
H_{p, q}^{m, n}\left[x \mid \begin{array}{l}
\left(a_{i}, \alpha_{i}\right)_{i=1, \ldots, p} \\
\left(b_{j}, \beta_{j}\right)_{j=1, ., q}
\end{array}\right]=\frac{1}{x^{c}} H_{p, q}^{m, n}\left[x \mid \begin{array}{c}
\left(a_{i}+c \alpha_{i}, \alpha_{i}\right)_{i=1, \ldots, p} \\
\left(b_{j}+c \beta_{j}, \beta_{j}\right)_{j=1, . ., q}
\end{array}\right]
$$

for all $c \in \mathbb{R}$ (see Mathai and Saxena [20]). 
We specialize the previous result by keeping in mind formula (2.10) and the operator (3.4).

Corollary 1. For $v=1$, Theorem 1 says that

$$
\frac{\partial}{\partial t} \tilde{u}_{1}^{\gamma, \mu}=\frac{1}{\gamma^{2}}\left(\frac{\partial}{\partial x} x^{2-\gamma} \frac{\partial}{\partial x}-(\gamma \mu-1) \frac{\partial}{\partial x} x^{1-\gamma}\right) \tilde{u}_{1}^{\gamma, \mu}, \quad x>0, t>0, \gamma \neq 0
$$

where $\tilde{u}_{1}^{\gamma, \mu}=\tilde{g}_{\mu}^{\gamma}$ is the distribution of the GGP.

This is because $L_{t}^{1} \stackrel{\text { a.s. }}{=} t$. Indeed, for $v=1, L_{t}^{v}$ is the elementary subordinator (see [4]).

Proof. If $v=1$, then the equation (5.10) takes the form

$$
\Psi_{t}(\eta)=\mathscr{M}\left[\tilde{g}_{\mu}^{\gamma}(\cdot, t)\right](\eta)=\Gamma\left(\frac{\eta-1}{\gamma}+\mu\right) \frac{t^{\frac{\eta-1}{\gamma}}}{\Gamma(\mu)}, \quad \Re\{\eta\}>1-\gamma \mu
$$

where $\tilde{g}_{\mu}^{\gamma}(x, t)=g_{\mu}^{\gamma}\left(x, t^{1 / \gamma}\right)$. For $\gamma>0$, we perform the time derivative of (5.16) and obtain

$$
\begin{aligned}
\frac{\partial}{\partial t} \Psi_{t}(\eta) & =\frac{\eta-1}{\gamma} \Gamma\left(\frac{\eta-1}{\gamma}+\mu\right) t^{\frac{\eta-\gamma-1}{\gamma}} \\
& =\frac{\eta-1}{\gamma}\left(\frac{\eta-\gamma-1+\gamma \mu}{\gamma}\right) \Gamma\left(\frac{\eta-\gamma-1}{\gamma}+\mu\right) t^{\frac{\eta-\gamma-1}{\gamma}} \\
& =\frac{1}{\gamma^{2}}(\eta-1)(\eta-\gamma-1+\gamma \mu) \Psi_{t}(\eta-\gamma)
\end{aligned}
$$

which coincides with (5.14) and $\tilde{u}_{1}^{\gamma, \mu}(x, t)=\tilde{g}_{\mu}^{\gamma}(x, t), \gamma>0$. Similar calculation must be done for $\gamma<0$ and the proof is completed.

Corollary 2. Let us write $\tilde{u}_{v}^{\mu}(x, t)=\tilde{u}_{v}^{1, \mu}(x, t)$. The distribution $\tilde{u}_{v}^{\mu}(x, t), x>0, t>0 \mu>0$, $v \in(0,1]$, solves the following fractional equation

$$
\frac{\partial^{v}}{\partial t^{v}} u_{v}^{\mu}=\left(x \frac{\partial^{2}}{\partial x^{2}}-(\mu-2) \frac{\partial}{\partial x}\right) u_{v}^{\mu}
$$

In particular, for $v=1 / 2$, we have

$$
\tilde{u}_{1 / 2}^{\mu}(x, t)=\frac{x^{\mu-1}}{\sqrt{\pi t} \Gamma(\mu)} \int_{0}^{\infty} s^{-\mu} \exp \left\{-\frac{x}{s}-\frac{s^{2}}{4 t}\right\} d s, \quad x>0, t>0, \mu>0
$$

which can be seen as the distribution of the process $\left\{G_{|B(2 t)|}^{1, \mu}, \quad t>0\right\}$ where $B$ is a standard Brownian motion run at twice its usual speed and $G_{t}^{\gamma, \mu}$ is a GGP. We notice that the process $G_{t}^{1, \mu}$ is a squared Bessel process starting from zero.

Corollary 3. The distribution $\tilde{u}_{v}^{2, \mu}=\tilde{u}_{v}^{2, \mu}(x, t), x>0, t>0, \mu>0, v \in(0,1]$ solves the following fractional equation

$$
\frac{\partial^{v}}{\partial t^{v}} \tilde{u}_{v}^{2, \mu}=\frac{1}{2^{2}}\left(\frac{\partial^{2}}{\partial x^{2}}-\frac{\partial}{\partial x} \frac{(2 \mu-1)}{x}\right) \tilde{u}_{v}^{2, \mu}
$$


In particular, for $v=1 / 3$, we have

$$
\tilde{u}_{1 / 3}^{2, \mu}(x, t)=\frac{2 x^{2 \mu-1}}{\pi \Gamma(\mu) \sqrt{t}} \int_{0}^{\infty} \frac{e^{-\frac{x^{2}}{s}}}{s^{\mu-1 / 2}} K_{\frac{1}{3}}\left(\frac{2}{3^{3 / 2}} \frac{s^{3 / 2}}{\sqrt{t}}\right) d s, \quad x>0, t>0, \mu>0
$$

and for $\mu=1 / 2$ we obtain

$$
\tilde{u}_{1 / 3}^{2,1 / 2}(x, t)=\frac{2}{\pi^{3 / 2} \sqrt{t}} \int_{0}^{\infty} e^{-\frac{x^{2}}{s}} K_{\frac{1}{3}}\left(\frac{2}{3^{3 / 2}} \frac{s^{3 / 2}}{\sqrt{t}}\right) d s, \quad x>0, t>0
$$

which is the distribution of $\left|B\left(L_{t}^{1 / 3}\right)\right|$ where $|B(t)|$ is a folded Brownian motion with variance $t / 2$.

Acknowledgement The author is grateful to the anonymous referee for careful checks and comments.

\section{References}

1 B. Baeumer and M. Meerschaert. Stochastic solutions for fractional cauchy problems. Fract. Calc. Appl. Anal., 4(4):481 - 500, 2001. MR1874479

2 B. Baeumer, M. Meerschaert, and E. Nane. Space-time duality for fractional diffusion. J. Appl. Probab., 46:1100 - 1115, 2009. MR2582709

3 L. Beghin and E. Orsingher. The telegraph process stopped at stable-distributed times and its connection with the fractional telegraph equation. Fract. Calc. Appl. Anal., 6:187 - 204, 2003. MR2035414

4 J. Bertoin. Lévy Processes. Cambridge University Press, 1996. MR1406564

5 L. Bondesson, G. K. Kristiansen, and F. W. Steutel. Infinite divisibility of random variables and their integer parts. Stat. Prob. Lett, 28:271 - 278, 1996. MR1407001

6 R. D. DeBlassie. Higher order PDEs and symmetric stable process. Probab. Theory Rel. Fields, 129:495 - 536, 2004. MR2078980

7 M. D'Ovidio and E. Orsingher. Composition of processes and related partial differential equations. J. Theor. Probab. (published on line), 2010.

8 M. D'Ovidio and E. Orsingher. Bessel processes and hyperbolic Brownian motions stopped at different random times. Under revision for Stochastic Processes and their Applications, (arXiv:1003.6085v1), 2010.

9 W. Feller. An introduction to probability theory and its applications, volume 2. 2 edition, 1971.

10 C. Fox. The G and H functions as symmetrical Fourier kernels. Trans. Amer. Math. Soc., 98:395 - 429, 1961. MR0131578

11 R. Gorenflo and F. Mainardi. Fractional calculus: integral and differential equations of frational order, in A. Carpinteri and F. Mainardi (Editors). Fractals and Fractional Calculus in Continuum Mechanics, pages 223 - 276, 1997. Wien and New York, Springher Verlag. MR1611585 
12 I. S. Gradshteyn and I. M. Ryzhik. Table of integrals, series and products. Academic Press, 2007. Seventh edition. MR2360010

13 A. Kilbas, H. Srivastava, and J. Trujillo. Theory and applications of fractional differential equations (North-Holland Mathematics Studies), volume 204. Elsevier, Amsterdam, 2006. MR2218073

14 A. N. Kochubei. The Cauchy problem for evolution equations of fractional order. Differential Equations, 25:967 - 974, 1989. MR1014153

15 A. N. Kochubei. Diffusion of fractional order. Lecture Notes in Physics, 26:485 - 492, 1990. MR1061448

16 S. Kotz, N. Balakrishnan, and N. L. Johnson. Continuous multivariate distributions, volume 1. New York, Wiley, 2nd edition, 2000. MR1788152

17 F. Mainardi, Y. Luchko, and G. Pagnini. The fundamental solution of the space-time fractional diffusion equation. Fract. Calc. Appl. Anal., 4(2):153 - 192, 2001. MR1829592

18 F. Mainardi, G. Pagnini, and R. Gorenflo. Mellin transform and subordination laws in fractional diffusion processes. Fract. Calc. Appl. Anal., 6(4):441 - 459, 2003. MR2044309

19 F. Mainardi, G. Pagnini, and R. Gorenflo. Some aspects of fractional diffusion equations of single and distributed order. Applied Mathematics and Computing, 187:295 - 305, 2007. MR2323582

20 A. Mathai and R. Saxena. Generalized Hypergeometric functions with applications in statistics and physical sciences. Lecture Notes in Mathematics, n. 348, 1973. MR0463524

21 M. Meerschaert and H. P. Scheffler. Triangular array limits for continuous time random walks. Stoch. Proc. Appl., 118:1606 - 1633, 2008. MR2442372

22 M. Meerschaert, E. Nane, and P. Vellaisamy. Fractional cauchy problems on bounded domains. Ann. of Probab., 37(3):979 - 1007, 2009. MR2537547

23 E. Nane. Higher order PDE's and iterated processes . Trans. Amer. Math. Soc., (5):681-692, 2008. MR2373329

24 E. Nane. Fractional cauchy problems on bounded domains: survey of recent results. arXiv:1004.1577v1, 2010.

25 R. Nigmatullin. The realization of the generalized transfer in a medium with fractal geometry. Phys. Status Solidi B, 133:425 - 430, 1986.

26 E. Orsingher and L. Beghin. Time-fractional telegraph equations and telegraph processes with Brownian time. Probab. Theory Rel. Fields, 128(1):141 - 160, 2004. MR2027298

27 E. Orsingher and L. Beghin. Fractional diffusion equations and processes with randomly varying time. Ann. Probab., 37:206 - 249, 2009. MR2489164

28 S. Samko, A. A. Kilbas, and O. I. Marichev. Fractional Integrals and Derivatives: Theory and Applications. Gordon and Breach, Newark, N. J., 1993. MR1347689 
29 W. Schneider and W. Wyss. Fractional diffusion and wave equations. J. Math. Phys., 30:134 144, 1989. MR0974464

30 W. Wyss. The fractional diffusion equations. J. Math. Phys., 27:2782 - 2785, 1986. MR0861345

31 G. Zaslavsky. Fractional kinetic equation for Hamiltonian chaos. Phys. D, 76:110 - 122, 1994. MR1295881

32 V. M. Zolotarev. One-dimensional stable distributions, volume 65 of Translations of Mathematical Monographs. American Mathematical Society, 1986. ISBN 0-8218-4519-5. Translated from the Russian by H. H. McFaden, Translation edited by Ben Silver. MR0854867 